\title{
Animal Monitoring with Unmanned Aerial Vehicle-Aided Wireless Sensor Networks
}

\author{
Jun Xu, Gürkan Solmaz, Rouhollah Rahmatizadeh, Damla Turgut and Ladislau Bölöni \\ Department of Electrical Engineering and Computer Science \\ University of Central Florida, Orlando, FL \\ Email: \{junxu,gsolmaz,rrahmati,turgut,lboloni\}@eecs.ucf.edu
}

\begin{abstract}
In this paper, we focus on an application of wireless sensor networks (WSNs) with unmanned aerial vehicle (UAV). The aim of the application is to detect the locations of endangered species in large-scale wildlife areas or monitor movement of animals without any attachment devices. We first define the mathematical model of the animal monitoring problem in terms of the value of information (VoI) and rewards. We design a network model including clusters of sensor nodes and a single UAV that acts as a mobile sink and visits the clusters. We propose a path planning approach based on a Markov decision process (MDP) model that maximizes the VoI while reducing message delays. We used real-world movement dataset of zebras. Simulation results show that our approach outperforms greedy and random heuristics as well as the path planning based on the solution of the traveling salesman problem.
\end{abstract}

Index Terms - value of information; unmanned aerial vehicle; UAV; animal monitoring; path planning; MDP.

\section{INTRODUCTION}

Wireless sensor networks (WSNs) have been applied to real-world habitat and environmental monitoring and created enormous potential benefits to society [1]. Currently, wide range of WSN applications exist for various environmental monitoring purposes such as forest fire monitoring [2], agricultural monitoring [3] and specific purposes such as volcanic eruption monitoring [4]. In some application scenarios of WSNs, such as forest fire monitoring or animal monitoring, the sensed information is time-sensitive. In other words, the earlier the information is reported to a sink or a base station, the higher importance the information has.

In this paper, we focus on the challenge of animal monitoring in wildlife using WSNs. Monitoring animals may have various goals such as tracking their migration paths or finding if specific endangered species exist in a region. Wildlife monitoring can be achieved by mounting devices on various species (e.g., cranes [5], zebras [6]). On the other hand, mounting sensor devices may have harmful effects on animals such as the unbalancing effect on birds [5]. Moreover, mounting devices is not feasible if the aim is to find whether some species exist. Our network model consists of static sensor nodes placed in strategic places that are empirically known as visited before by the monitored animals (e.g., places with water supply).

The UAV systems are cost effective and attractive solutions for surveillance applications. We consider UAVs participating in animal monitoring operation as sinks which collect time sensitive information from the sensor devices and also directly observe and track the animals.

In our application scenario, we consider large wildlife areas which we call the observation areas. An observation area is uniformly divided into virtual grids. Each grid includes a cluster of sensor nodes and a clusterhead is chosen among them. The clusterhead is responsible for receiving data packages from the sensor nodes via hop-by-hop wireless communication and sending them to a UAV. The UAV acts as a mobile sink and autonomously visits clusters of sensor nodes in distinct regions of the observation area for gathering data from clusterheads.

By separating the WSN into clusters of sensor nodes in distinct regions, data collection of the UAV reduces to visiting the grids with cluster heads. In other words, for gathering data, the UAV will move from one cluster to another during its operation. In such a network setting, performance of the WSN highly depends on the movement choices of the UAV. For instance, if the UAV visits the regions with critical information earlier than the other regions, the WSN can achieve higher value of information (VoI) [7], [8], lower data losses, and decreased message delays.

We define a mathematical model for calculating the value of information in animal monitoring operation and use it for decision making of visiting clusters by the UAV. We define a Markov decision process (MDP) model for the UAV such that states represent the grids and actions lead to deterministic state transitions. We propose a Markovian decision-based path planning approach for efficient data collection using single a UAV. In this approach, while actions are chosen to visit states with higher possible rewards, there is a probability of taking a random action just to explore the area in each movement decision of the UAV. Even though we focus on path planning of single UAV in this papers, our mathematical model can be used for collaborative data collection approaches using multiple UAVs and can be extended path planning algorithms.

We evaluate the performance of the proposed network model and the path planning approach. ZebraNet [6] dataset that includes the GPS traces of zebras is used as a basis for the network simulation. Markovian decision-based path planning approach is compared against greedy and random heuristics as well as the traveling salesman problem (TSP) solution. We observe that the proposed approach outperforms other approaches in terms of VoI, average message delay, and the number of directly detected zebras. 


\section{RELATED WORK}

Akbas et al. [9] propose a wireless sensor and actor network (WSAN) protocol for analyzing the social network of an ape society through mobility. In their experiments, each gorilla is equipped with a wireless sensor node while the silverbacks are equipped with actors. Tuna et al. [10] propose using UAVs for deployment of sensor nodes for post-disaster monitoring. Our study differs from the aforementioned ones as we propose using UAV as a major element of the WSNs for monitoring purpose.

UAVs are used as sensor nodes for monitoring various species in nature. Hodgson et al. [11] use ScanEagle UAV to survey marine mammals. Their results indicate that UAVs are not limited by sea state conditions as sightings from manned surveys. Chamoso et al. [12] propose using UAVs for scanning large areas of livestock systems. Using visual recognition techniques, the recorded images are used to count and monitor animal species. Brust et al. [13] propose a swarm model for multiple UAVs on reliable forest mapping. Swarm connectivity and communication are maintained under their network structure. In our model, we propose using UAV as a mobile sink for data collection.

Efficient use of mobile sinks in WSNs is a well-investigated problem. Mobile sinks provide some advantages such as distributing the energy consumption throughout the network and increase network lifetimes. Rahmatizadeh et al. [14] study sink mobility in virtual coordinates domain. Solmaz et al. [15], [16] propose positioning approaches for multiple mobile sinks to optimize event coverage in WSNs. Basagni et al. [17] investigate the problem of maximizing value of information in underwater sensor networks. They formulate the problem using an Integer Linear Programming (ILP) model for path planning of underwater vehicles. Their method achieves better results in terms of VoI compared to a greedy heuristic. Our sink mobility approach is different from these models as the main objective of our path planning approach is to maximize VoI using an unmanned aerial vehicle.

\section{Mathematical Model}

In this section, we describe the mathematical model of the value of information for animal monitoring and our definition of the sensors' credibility and initial rewards.

\section{A. Value of information}

The value of information ( $\mathrm{VoI})$ is a metric initially proposed in game theory as the price an optimal player would pay for a piece of information. This metric has been redefined for sensor networks by Turgut and Bölöni [7], [8] by assigning to the data the value of the optimal action enabled by it. As late actions are usually less valuable, the VoI of an event is normally highest at the moment the event is created and decreases as time passes.

Let us describe an example environmental mission where VoI has critical importance. In the times of marine oil spills, where crude oil is released into ocean or coastal waters from offshore platforms or tankers, actions must be taken to stop leakage and repair the damages at the earliest time. After the oil leakage information is sensed by a set of underwater sensor nodes, early arrival of the information to the base station will give the operators ability to make more timely decisions for repairing and patching up the leaks.

To the best of our knowledge, the concept of $\mathrm{VoI}$ is not considered for the animal monitoring problem in the literature. However, maximizing VoI may be very useful for monitoring wild animals such as finding the current locations of endangered species. For instance, earlier arrival of the information to a UAV that collects data may be helpful for finding the exact location of an animal in need of rescue. Moreover, earlier information retrieval by the UAV may result in direct observation of the animal.

The idea behind VoI can be described by a scenario where an actuation action must be taken on the basis of sensed data. The sensed information is of more value at present as compared to being processed for actuation at a later time. For many scenarios, we define the value of information in terms of an exponential decay (although other forms are possible):

$$
F_{V o I}(t)=A e^{-B t}
$$

In Equation 1, the constant value $\mathrm{A}$ represents the initial value of the information while $\mathrm{B}$ represents the decay speed of the VoI. A higher value of A defines the information with a higher initial value while a higher value of $B$ defines a faster decay of the VoI. Figure 1 shows three different examples of $\mathrm{A}$ and $\mathrm{B}$ values and their outcomes as the function of VoI. It may be considered as representations of three urgent levels of data in a sensor network. As it can be seen in the figure, higher values such as $A=10$ and $B=0.1$ produce the VoI with sharper decays, meaning urgent events that lose their importance earlier. If we define a threshold value of events as $F_{V o I}=1$, the events with higher values of $\mathrm{A}$ and $\mathrm{B}$ expire before 60 minutes while the events with lower values $(A=5$, $B=0.02$ ) expire around 90 minutes.

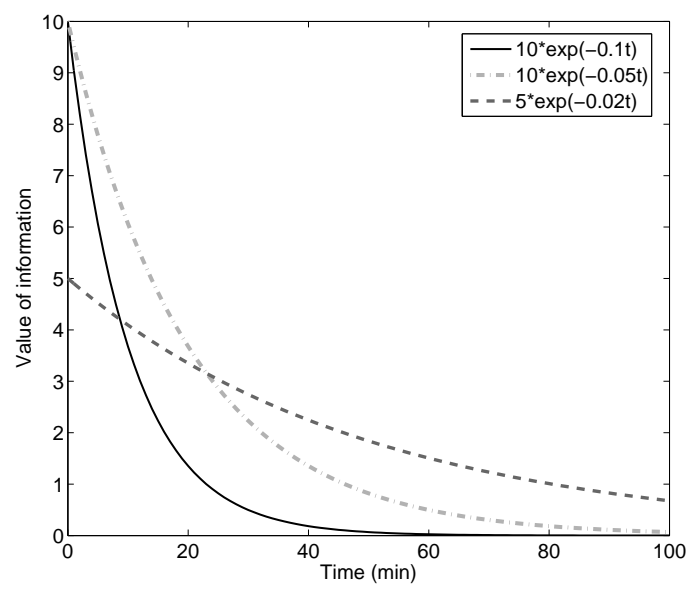

Fig. 1. VoI for different A and B values. 


\section{B. Initial rewards}

We define each sensed information as an event in the system. Each event has an initial reward (IR) that is the value of information at the time of the event occurs $(t=0)$. In other words, IR is the maximum value of information that can be gathered for each detected event. In our problem, IR is affected by several factors: credibility of sensors, distance to the animal and the duration.

Credibility $(\mathrm{C})$ is a parameter that represents the reliability of the sensed information. We define credibility based on the heterogeneous sensor nodes with different abilities and quality of services. For instance, an acoustic sensor node detects the sounds coming from an animal while another sensor node can take visual images. Moreover, a sensor node may have less capable camera that results in lower resolution images than the other sensor nodes. We assume there are $k$ sensor nodes $\left\{N_{1}, N_{2}, \ldots, N_{k}\right\}$ deployed in the target area. Different types of sensor nodes perceive different types of information such as picture, sound, and odor. Each data type has a weight value according to its importance $\left\{W_{1}, W_{2}, \ldots, W_{k}\right\}$. We consider quality and resolution of the data as a factor of credibility. Images taken by cameras with different resolutions and images with different brightness and contrast levels (e.g, an image taken at night and another in the sunlight) can be given as such examples.

Let us define the credibility for the $i^{t h}$ event as

$$
C_{i}=\lambda \times W_{i}
$$

where $\lambda$ is an impact factor of the quality and resolution of the sensed data. We consider the maximum $C_{i}=1$ with $W_{i}=1$, $\lambda=1$, and the minimum $C_{i}$ to be equal to 0 .

The distance of an animal to the sensor node is considered as an important factor of the initial reward. The main reason is its effect to the accuracy of the data. For instance, the loudness of sounds from the animals may affect the accuracy of classification of the animal species. Another example may be the resolution of the image taken by a sensor node or the distance of the image.

$$
I_{d i s t}=\alpha \times \frac{1}{A_{e s t}}
$$

By Equation 3, distance to the animal will be reflected into an estimation of the area size where the animal is located. The smaller the estimated area $A_{e s t}$ is, the more credible the sensing result is. $\alpha$ is a constant value used for adjusting the value $I_{\text {dist }}$.

We define the duration parameter $I_{\text {duration }}$ of the sensed event for the initial reward. Events having longer durations are considered more effective evidences for the appearances of the animals. For instance, an event with a longer duration may infer that the animal prefers staying in the proximity of the sensor node. $I_{\text {duration }}$ also used for data types such that when an animal is detected, sensor nodes records series of images with a previously defined frequency. Some environmental noise may also cause similar results. We consider a threshold value $T_{\max }$ for the maximum duration that leads to ideal $I_{\text {duration }}$ and define the duration parameter as

$$
I_{\text {duration }}=\frac{T}{T_{\max }}
$$

where $T$ is the duration of the event and $0<I_{\text {duration }} \leq 1$.

Finally, we define the initial reward $I R_{i}$ of the event $i$ as below.

$$
I R_{i}=\sigma \times C_{i} \times I_{\text {dist }} \times I_{\text {duration }}
$$

where $\sigma$ is a parameter that depends on the type of animal. For instance, information sensed by endangered animal species may have better reward.

$V o I_{i}$ shows the value of the sensed event $i$ at the time when it is sent to a mobile sink (UAV). $V o I_{i}$ has the maximum value at the moment the event is detected and then it gradually decreases as time passes.

$$
V o I_{i}=I R_{i} \times e^{-B t}
$$

where B is a factor to control the convergence speed of the VoI. Our main objective is to maximize VoI collected by the UAV during its operation.

\section{Animal Monitoring System}

\section{A. Network model}

Considering an observation area with animals living in it, the goal is to monitor specific animals' appearance. In our approach, we divide the area into virtual grids. In each grid, a set of sensor nodes with monitoring functionalities are deployed.

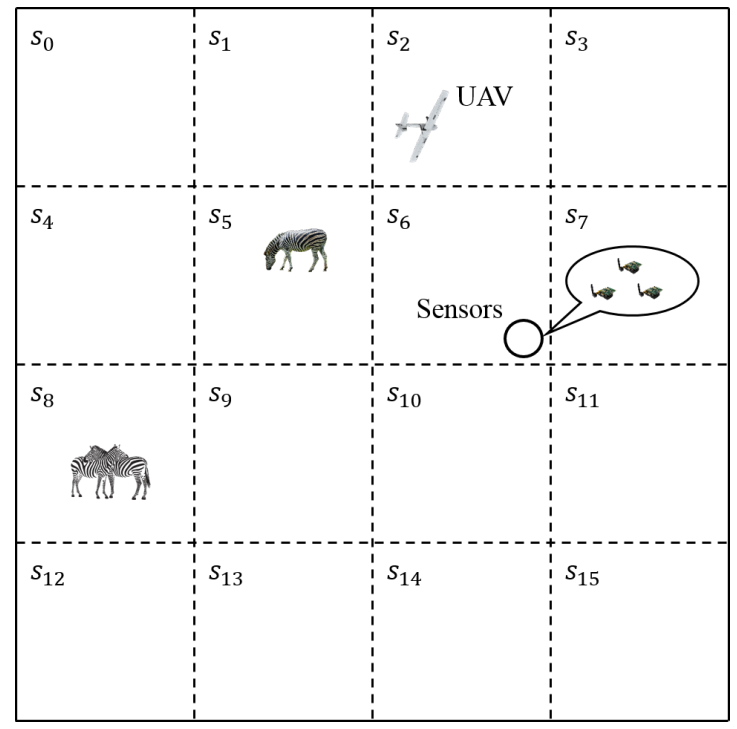

Fig. 2. The network model. 
1) Sensor nodes: Sensor nodes are deployed by uniform random distribution in strategic parts of the observation area. As illustrated in Fig. 2, sensors in a grid can be treated as a cluster and a clusterhead is selected periodically. Sensors inside a virtual grid can communicate with the clusterhead directly or via a hop-by-hop communication. The clusterhead is responsible for receiving event messages from other sensors and then submitting all the event messages to the UAV. Routing and clustering are not within the scope of this paper as they are well-investigated research topics.

2) Unmanned aerial vehicles: UAVs have been widely used in various applications due to their advantages such as flexibility, fast speed and good endurance. In this WSN application, UAV is used as an autonomous mobile sink for gathering time sensitive information. Apart from previously mentioned advantages, using UAV for animal monitoring not only overcomes the geographical challenge but also has no harmful effects on animals such as the unbalancing effect [5].

Assumptions of the network model are given as follows:

- There is a single UAV

- The UAV has no energy constraints, while it is not the case for the sensor nodes.

- The UAV flies with a fixed speed and it only communicates with clusterheads.

\section{B. Markovian decision-based path planning}

Wildlife animals have their own habitats, which means they are more likely to stay at a certain location for rest or just having activities in a small area. Fig. 3 shows real movement trajectories of 4 zebras in 3 days.

In this figure, the regions visited by the zebras are marked with their corresponding grid indices. As it can be seen, the mobility choices of zebras are not random. Instead, most of the time they prefer to stay in the places shown with gray color and they appear in other marked regions in shorter durations. For instance, in the top-left figure, a zebra spends most of the time in 5 out of 16 regions $\left(s_{1}, s_{4}, s_{5}, s_{6}, s_{1} 3\right)$ while shortly passing through the regions $s_{1} 2, s_{8}, s_{9}$, and $s_{0}$. Furthermore, we observe that a zebra can visit an already visited location multiple times as the regions $s_{0}$ and $s_{1}$ were visited by another zebra in the bottom-right figure. We conclude that sensing a zebra in a region may infer the possibility of future visits to the same region.

Based on these observations, we use a Markov decision process (MDP) model for the path planning problem of UAV. A finite state MDP is defined with a 5-tuple $(S, A, P, D, R, \gamma)$ where $S=s_{0}, s_{1}, \ldots, s_{m}$ is a finite set of states. $A$ is a set of actions and $P$ is a set of state transition probabilities. $D$ is the initial-state distribution, from which the start state $s_{0}$ is drawn. $R: S \longrightarrow A$ is the reward function and $\gamma \in[0,1)$ is a discount factor. In the context of UAV path planning, we define the elements of MDP as follows.

- $S$ is the set of states (grids) in the network.

- $A$ is the set of cardinal directions that UAV can go plus staying in the same grid: \{north, east, south, west, northeast, southeast, southwest, northwest, stay $\}$.
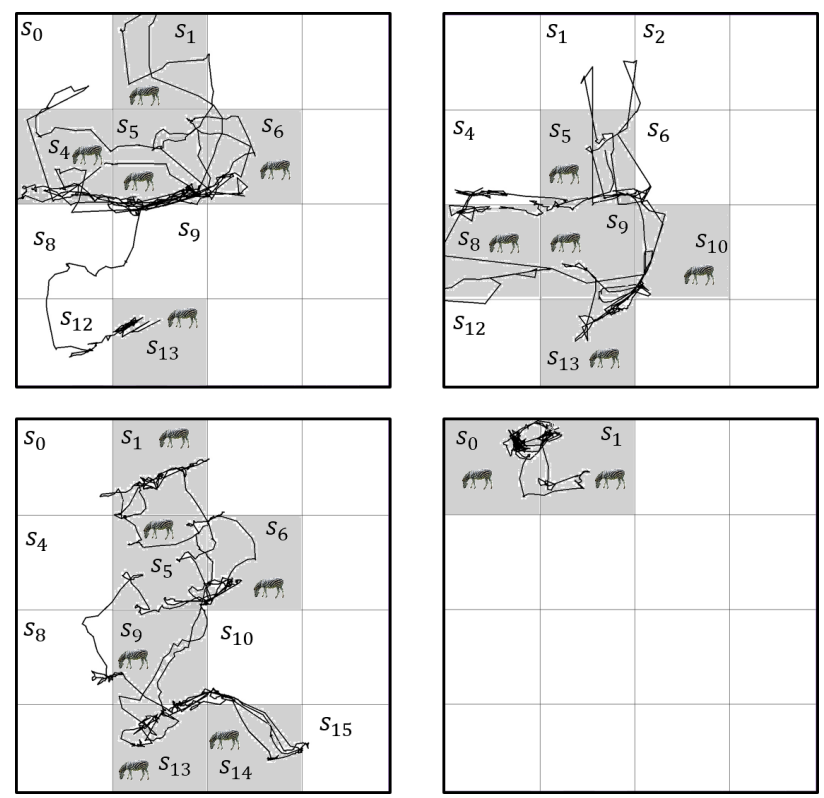

Fig. 3. Movement trajectories of 4 zebras from the ZebraNet dataset.

- $P$ is the set of state transition probabilities. Our model is deterministic, i.e. the probability of ending up in the desired grid is 1 . The probability of accidentally appearing in non-desired states is 0 .

- $D$ is the initial-state distribution which is 1 for the top-left corner grid in the network and 0 for all other grids. This means that the algorithm always starts from state $s_{0}$.

- $R$ is the reward UAV gets if it enters gird $s$. Reward is calculated using Equation 5.

- $\gamma \in[0,1)$ is the discount that obtaining the information in distant future worth less compared to getting informed in the near future.

In our model, the set of clusterheads in the network are represented by the set of states $S$ in MDP. In this setting, UAV needs to make decision on its next visiting location after collecting the clusterhead's event messages, which can be represented by the state transitions in the MDP model. After visiting each clusterhead, the UAV will update its information of the network and decide the new visiting clusterhead. This decision process has an analogy to the MDP in which rewards of the previous actions will have an effect on the next state transition decision. Lastly, maximizing the VoI in our problem means optimizing rewards in MDP.

We introduce MDP modeling into our path planning problem. Solving the MDP gives a policy which tells us the next grid in the observation area that the UAV should visit. To solve the MDP, we use Q-learning algorithm [18]. By utilizing value iteration method, we calculate the Q-value for each state using the following equation.

$$
Q(s, a) \leftarrow R(s)+\gamma \max _{a^{\prime}} Q\left(s^{\prime}, a^{\prime}\right)
$$

$Q(s, a)$ is the new Q-value for taking action $a$ when the 


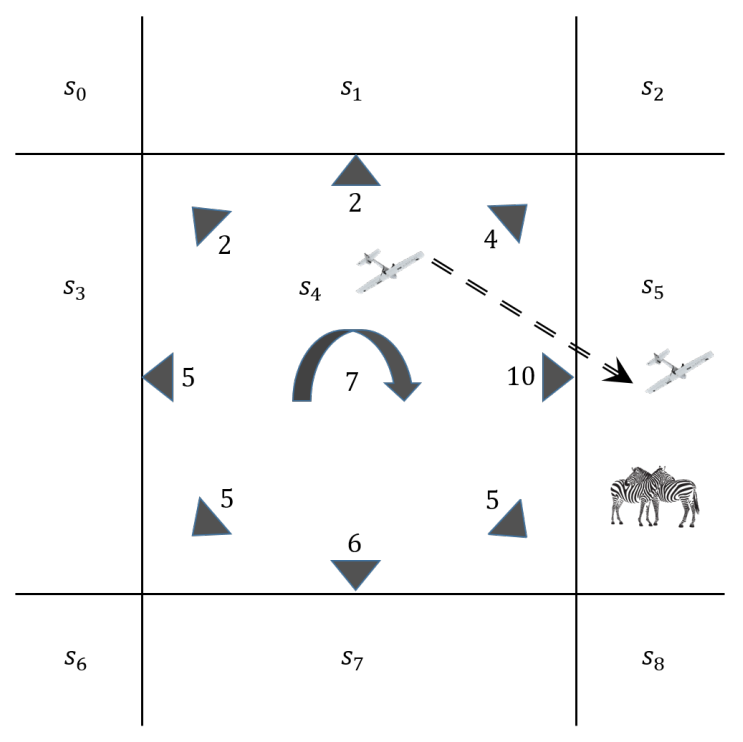

Fig. 4. Deciding the next state based on Q-values.

UAV is in state $s . R(s)$ is calculated using Equation 5. $s^{\prime}$ is the grid the UAV appears when it takes action $a$ and $a^{\prime}$ is any action possible when we are in state $s^{\prime}$. This means each time $a$ is taken from state $s$, UAV reaches state $s^{\prime}$, calculates the maximum Q-value of the next state by taking any arbitrary action from state $s^{\prime}$, sums it with initial reward it gets as it enters state $s^{\prime}$, and uses this value to update $Q(s, a)$.

In the context of UAV path planning, when the UAV goes to one of the neighboring grids, it may find zebras or may not find anything. The UAV will then update the reward of coming to this state from the previous state. The outcomes of these visits help the UAV to decide which grid to visit next. Formally, this policy is obtained using the following equation.

$$
\pi(s)=\arg \max _{a} Q(s, a)
$$

Fig. 4 illustrates path planning decision for the UAV based on the Q-value of each action in the current state. The UAV is initially in state $s_{4}$ as it decides which of the adjacent states it should visit. The actions are shown by the arrows. Available actions are either visiting the neighboring states or staying in the same state. The Q-value corresponding to each action is shown near each arrow. The UAV decides to take action which leads to state $s_{5}$ since the Q-value of the corresponding action is higher than the others. In addition, there is a probability of random exploration during selection of the next state. The exploration is based on the $\epsilon$-greedy policy of Q-learning.

The flow-chart of the proposed path planning approach can be seen in Fig. 5. Both exploration and exploitation are important for the UAV performance. After visiting each clusterhead, the UAV needs to select the next grid with $\epsilon-$ greedy policy, $0<\epsilon<1$. The UAV selects the next grid in a stochastic way with probability $\epsilon$; otherwise, it visits the next state in a deterministic way of choosing the best action.

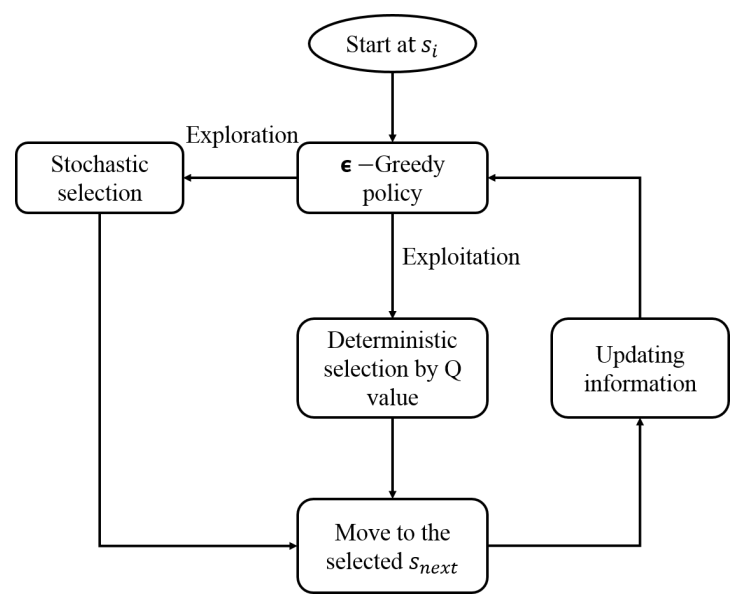

Fig. 5. Flow chart of state transitions of the UAV.

\section{Simulation Study}

\section{A. Simulation environment}

The proposed WSN application and the UAV path planning approach are tested with simulation experiments. We implemented a Java-based discrete time simulator. The movement traces of zebras come from the ZebraNet [6] dataset that contains the location information of 5 zebras in June 2005 at a $10 \mathrm{~km} \times 10 \mathrm{~km}$ area near Nanyuki, Kenya. The sampling time of the GPS traces is 1 minute and the total experiment time is 5 days. We establish our experimental network by converting the information from the dataset into our grid-based network model. A single UAV is used for event message collection.

The purpose of the application is to detect the appearances of the zebras. During the operation, when a zebra first appears in a region, the sensor nodes should record it. However, if the same zebra stays in a small area for a long time, such as the case when the zebra sleeps there, it may cause excessive energy consumption to the sensor nodes. Therefore, we define the events as following.

- If a zebra moves from one grid to another, we record it as an event.

- If a zebra always stays in a grid, instead of recording every minute (the sampling time interval of the data), we periodically record its location once in every $\Delta t$ amount of time.

Considering the proposed WSN model, the UAV needs to collect messages from sensors deployed on the ground. As a result, the speed and the altitude of UAV should not be very high. In this case, we used a Bayraktar mini UAV [19] for our experiments since it has a good endurance performance and a moderate maximum flight speed $(60 \mathrm{~km} / \mathrm{h})$.

Using 1 minute sampling time as the experimental unit time causes the UAV to move $1 \mathrm{~km}$ each time step that produces low resolution results. To make the results more accurate, we set the unit experimental time steps (rounds as 10s). In this case, the UAV speed is set as $100-200 \mathrm{~m} /$ round.

Table I includes the parameter values used in our experiments. While we assume homogeneous events for simplicity in 
TABLE I

EXPERIMENTAL PARAMETERS

\begin{tabular}{|l|l|}
\hline Network size & $10 \mathrm{~km} \times 10 \mathrm{~km}$ \\
Number of grids (states) & 16 \\
Grid size & $2500 \mathrm{~m} \times 2500 \mathrm{~m}$ \\
Unit experimental time (round) & $10 \mathrm{~s}$ \\
UAV speed & $100-200 \mathrm{~m} /$ round \\
Decay speed of VoI (parameter B) & 0.05 \\
Radius $r$ for direct observation & $200 \mathrm{~m}$ \\
Initial reward $I R\left(\sigma, C_{i}, I_{\text {dist }}, I_{\text {duration }}\right)$ & $10.0(10.0,1.0,1.0,1.0)$ \\
\hline
\end{tabular}

the simulation experiments, in real-world applications events have different IR values based on the mathematical model in Section III.

For the performance evaluation of the proposed Markovian decision process-based (MDP) path planning approach, we compare its outcomes with three other path planning approaches: greedy, traveling salesman problem-based (TSP) and random. All four approaches are used for autonomous movement of the UAV in the network for collection of event messages.

Let us briefly describe the three path planning approaches in the simulation study. As described in Section III, each event has an initial reward (IR). In the greedy approach, when the UAV visits a grid, IRs of events in this grid are summed for future grid selection. The UAV always pursues the highest local IR in its movement. In other words, the UAV flies to a neighbor grid with the highest IR. In addition, the UAV also maintains a visited grid list to guarantee all grids are visited. In the case of multiple neighbor grids having the same IRs, the UAV randomly selects one of them.

In the TSP approach, solution to the TSP problem is used as it provides the shortest path of visiting all grids among all the possible paths. Given the network structure, the path produced by TSP is actually fixed, which means the UAV always flies along a pre-set movement path. This pre-determined path feature results in a very stable experimental performance and also can be used as a good reference for comparing with adaptive approaches. Lastly, in the random approach, the UAV simply selects its next destination grid randomly among all the grids in the network.

To systematically examine the performance of a path planning strategy, we include results with three performance metrics in our simulation study.

1) Value of information: $\mathrm{VoI}$ is the most essential metric in animal monitoring application and maximizing the $\mathrm{VoI}$ is the main goal in designing the proposed MDP path planning approach. The definition of VoI is given in Section III and the parameter values used in the experiment is given in Table I.

2) Message delay: Since event messages can only be kept in sensors and wait in their buffer until being sent to the UAV, message delay is an important metric. Long message delays make the event messages lose their values. In this experiment, we measure the average message delay and the message delay distribution of all events.

3) Number of zebras encountered: As the UAV flies in the network, we define a radius $r$ inside which the zebras are assumed to be directly observed by the UAV. Although the number of zebras encountered is not the main goal in designing our path planning approach, direct encounters can be helpful for the monitoring as the UAV can capture higher resolution images.

\section{B. Simulation results}

Let us include the experimental results of the path planning approaches. We implemented 4 independent UAVs which are controlled by MDP, greedy, TSP and random approaches respectively. The results are obtained from an average of 10 simulation runs.

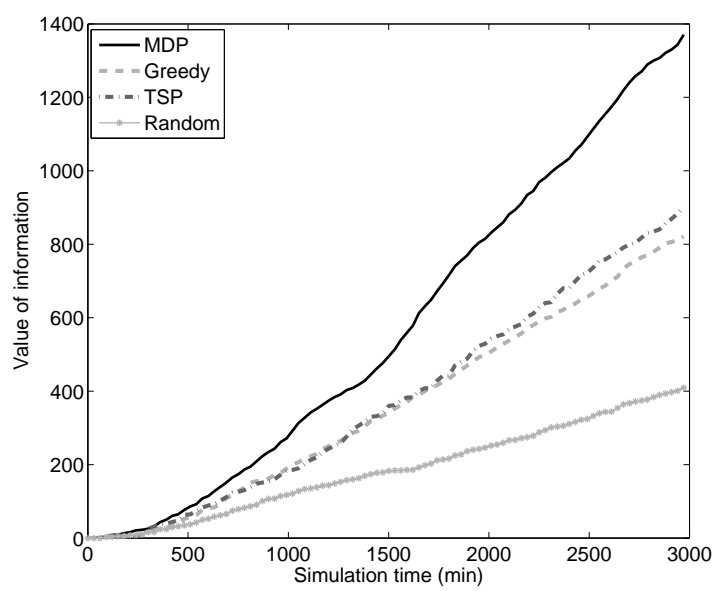

Fig. 6. Performance comparison of VoI w.r.t. simulation time.

Fig. 6 shows the performances of the path planning approaches in terms of the VoI w.r.t. simulation time. In MDP, the UAV continuously learn the appearance of zebras in the network and as a result it is more likely to visit grids that previously had zebras. It can be seen that MDP does not show a good performance at the beginning of the simulation since the learning process has just started and there is not much information in the Q-table. As the learning process continues, the VoI obtained by MDP gradually outperforms others. The random approach performs the worst due to its totally random state selection. The result of TSP is slightly better than greedy. This is because the greedy approach always selects the grid with the maximum IR from the neighbor grids. This local maximization policy limits its overall performance. With the TSP approach, the UAV always flies along a fixed path in the network, resulting in a stable performance.

Fig. 7 shows the impact of $\epsilon-$ greedy policy on the VoI. It can be seen that when $\epsilon=0.8$, which means the UAV has $80 \%$ probability to select the next state randomly, the performance is the worst. As the value of $\epsilon$ decreases from 0.8 to 0.4 , the probability of making deterministic decisions increases. This causes an improvement in the VoI performance. On the other hand, we also observe that when $\epsilon=0.2$ the performance of $\mathrm{VoI}$ is worse than that when $\epsilon=0.4$. This shows that the exploration is also important and the total deterministic 


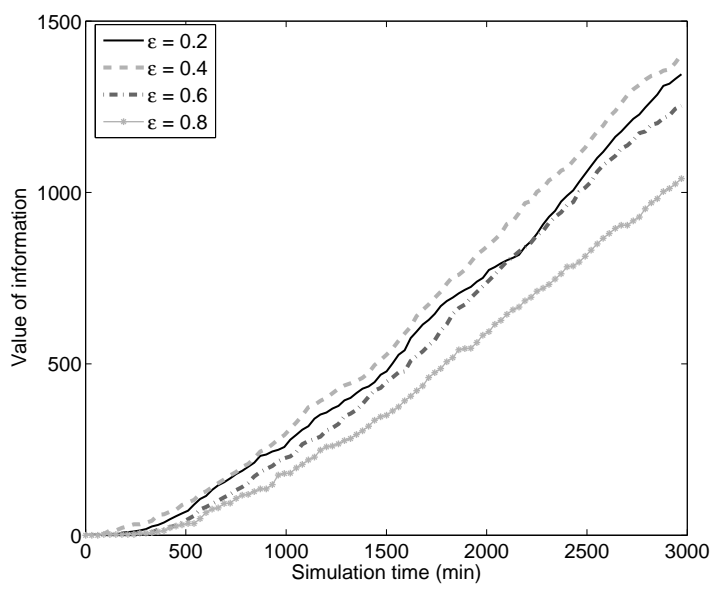

Fig. 7. The impact of $\epsilon$ on VoI w.r.t. simulation time.

policy can actually limit the performance. Empirically, we use $\epsilon=0.4$ in our experiments as it provides the best tradeoff.

The performance results of different simulation runs using the MDP approach are shown in Fig. 8. Note that the $\epsilon-$ greedy policy brings stochastic next state decisions and therefore leads to randomness in the results. To examine the stability of our approach despite the randomness, we compare 4 randomly selected simulation runs of the MDP approach with the same parameter settings. It can be seen that though some slight differences exist between results of the different simulation runs, the outcomes are not significantly different.

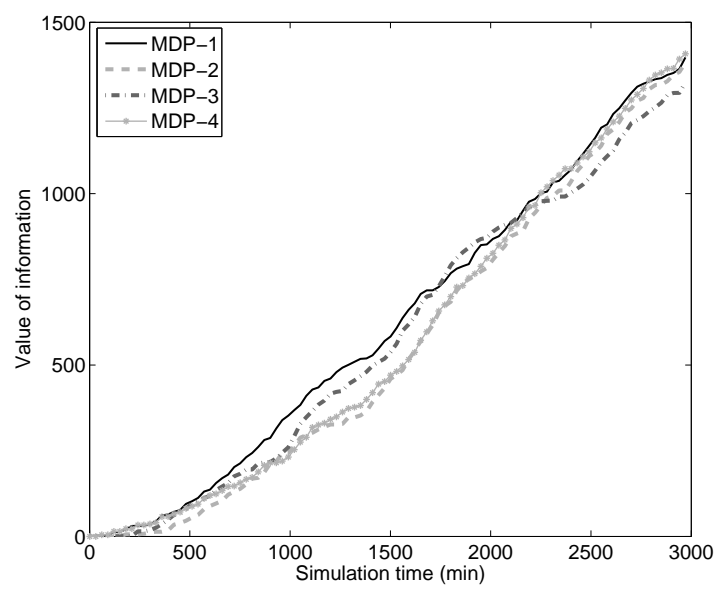

Fig. 8. Stable performance of MDP.

We include the results for the average message delay in Fig. 9. The standard errors show the stability of the path planning approaches in terms of message delay. It can be seen that the random approach has the highest average message delay, which is followed by the greedy one. Interestingly, we observe that the standard error of TSP is 0 . This is because TSP has a fixed path and the movement trajectories of the zebras are the same, which results in constant performance.

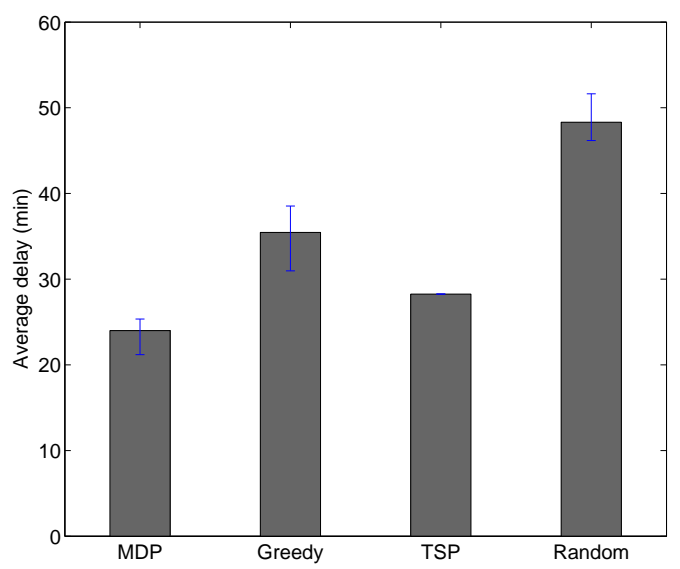

Fig. 9. Average message delays of the MDP, greedy, TSP, and random approaches.

Fig. 10 shows the probability distribution function (PDF) of the message delay for each path planning approach. As it can be seen, MDP is the clear winner with more than $40 \%$ of all messages having less than 5 minutes message delays. On the other hand, for greedy and TSP, only less than $25 \%$ of the same messages are gathered in less than 5 minutes. The random approach again has the worst performance resulting in less than $15 \%$ of the messages with short message delays.

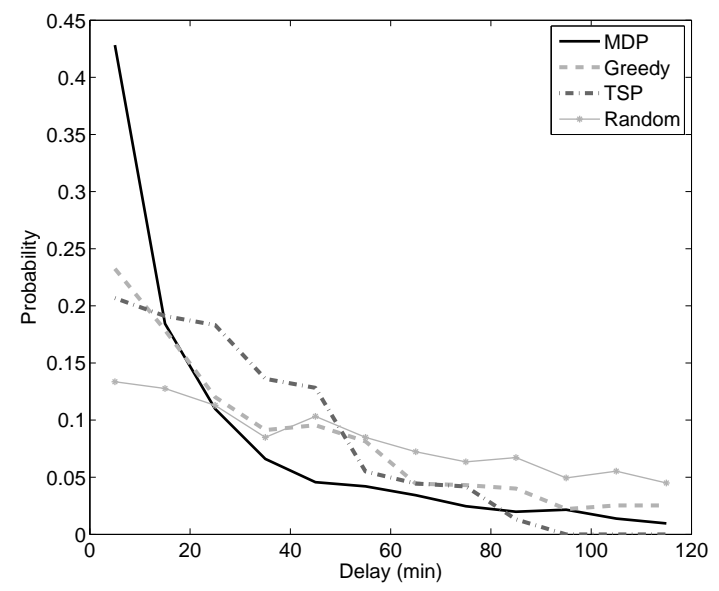

Fig. 10. PDF of message delays of the MDP, greedy, TSP, and random approaches.

Lastly, we analyze the results for the total number of zebras encountered by the UAV in Fig. 11. The random path planning approach causes the UAV to encounter the least number of zebras during its operation. While the greedy and TSP approaches seem to result in the same number of encountered zebras, TSP is more stable than greedy. This is because the UAV in greedy approach visits the grids randomly when the IRs of all neighbor grids are the same. Even though the MDP approach does not cause the UAV to track zebras directly, 
it still provides the best performance. This is an expected outcome of the model after a learning period, since the UAV has a high probability of visiting the grids where zebras have appeared before.

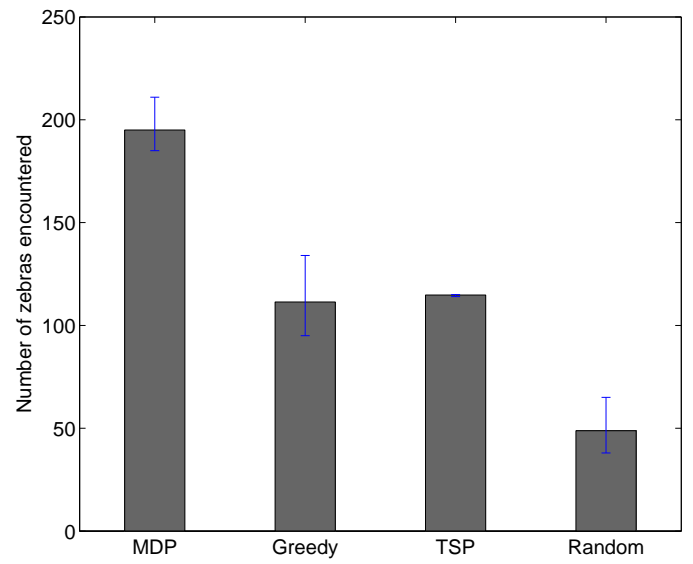

Fig. 11. Number of zebras encountered for the MDP, greedy, TSP, and random approaches.

Overall, it can be seen in the experimental results that MDP highly outperforms the other path planning approaches. Compared to the greedy approach as an example, MDP produces $80 \%$ increase in VoI, $30 \%$ increase in average message delay, and $90 \%$ increase in the number of zebras encountered. Although greedy path planning approach also relies on the previous information, it considers only the last time instance of zebra appearances in each grid. Unlike the greedy approach, the UAV in MDP continuously learn the events from the network and then update the Q-value in each grid. The Qtable maintained by the UAV reflects all previous information on the activities of zebras. As the learning process continues, the UAV is more likely to go to the hot-spots where zebras often appear. Additionally, both exploration and exploitation modes are introduced so that while the UAV is visiting the hot-spots, it also explores new regions in the observation area.

\section{CONCLUSION}

In this paper, we propose using UAV-aided WSNs for animal monitoring in wildlife areas. In our model, the UAV visits the clusters of static sensor nodes for data collection purpose. We propose a Markovian decision-based path planning approach for the UAV to maximize the VoI. The proposed network model is simulated using real-world mobility traces of zebras. Simulation results show that the performance of the proposed path planning approach is better than random, greedy, and TSP-based approaches. The MDP approach results in approximately $60 \%$ increase in $\mathrm{VoI}$ and the number of observed zebras and $20 \%$ decrease in message delays.

\section{REFERENCES}

[1] A. Mainwaring, D. Culler, J. Polastre, R. Szewczyk, and J. Anderson, "Wireless sensor networks for habitat monitoring," in Proc. of ACM WSNA'02 Workshop, September 2002, pp. 88-97.
[2] Y. E. Aslan, I. Korpeoglu, and Ö. Ulusoy, "A framework for use of wireless sensor networks in forest fire detection and monitoring," Computers, Environment and Urban Systems, vol. 36, no. 6, pp. 614625, November 2012.

[3] S. E. Díaz, J. C. Pérez, A. C. Mateos, M.-C. Marinescu, and B. B. Guerra, "A novel methodology for the monitoring of the agricultural production process based on wireless sensor networks," Computers and Electronics in Agriculture, vol. 76, no. 2, pp. 252-265, May 2011.

[4] M. İ. Akbaş and D. Turgut, "APAWSAN: actor positioning for aerial wireless sensor and actor networks," in Proc. of IEEE LCN'11, October 2011, pp. 567-574.

[5] D. Anthony, W. P. Bennett, M. C. Vuran, M. B. Dwyer, S. Elbaum, A. Lacy, M. Engels, and W. Wehtje, "Sensing through the continent: towards monitoring migratory birds using cellular sensor networks," in Proc. of ACM IPSN'12, April 2012, pp. 329-340.

[6] P. Juang, H. Oki, Y. Wang, M. Martonosi, L. S. Peh, and D. Rubenstein, "Energy-efficient computing for wildlife tracking: Design tradeoffs and early experiences with ZebraNet," SIGARCH Comput. Archit. News, vol. 30 , no. 5 , pp. 96-107, December 2002.

[7] D. Turgut and L. Bölöni, "A pragmatic value-of-information approach for intruder tracking sensor networks," in Proc. of IEEE ICC'12, June 2012, pp. 4931-4936.

[8] D. Turgut and L. Bölöni, "IVE: improving the value of information in energy-constrained intruder tracking sensor networks," in Proc. of IEEE ICC'13, June 2013, pp. 6360-6364.

[9] M. İ. Akbaş, M. R. Brust, C. H. Ribeiro, and D. Turgut, "fAPEbookanimal social life monitoring with wireless sensor and actor networks," in Proc. of IEEE GLOBECOM'10, December 2010, pp. 1-5.

[10] G. Tuna, T. V. Mumcu, K. Gulez, V. C. Gungor, and H. Erturk, "Unmanned aerial vehicle-aided wireless sensor network deployment system for post-disaster monitoring," Emerging Intelligent Computing Technology and Applications, vol. 304, pp. 298-305, July 2012.

[11] A. Hodgson, N. Kelly, and D. Peel, "Unmanned aerial vehicles (UAVs) for surveying marine fauna: a dugong case study," PloS one, vol. 8, no. 11, p. e79556, November 2013.

[12] P. Chamoso, W. Raveane, V. Parra, and A. González, "UAVs applied to the counting and monitoring of animals," Ambient Intelligence - Software and Applications, vol. 291, pp. 71-80, May 2014.

[13] M. R. Brust and B. M. Strimbu, "A networked swarm model for UAV deployment in the assessment of forest environments," in Proc. of IEEE ISSNIP'15, April 2015, pp. 1-6.

[14] R. Rahmatizadeh, S. A. Khan, A. P. Jayasumana, D. Turgut, and L. Bölöni, "Routing towards a mobile sink using virtual coordinates in a wireless sensor network," in Proc. of IEEE ICC'14, June 2014, pp. 12-17.

[15] G. Solmaz and D. Turgut, "Optimizing event coverage in theme parks," Wireless Networks (WINET) Journal, vol. 20, no. 6, pp. 1445-1459, August 2014.

[16] G. Solmaz, K. Akkaya, and D. Turgut, "Communication-constrained pcenter problem for event coverage in theme parks," in Proc. of IEEE GLOBECOM'14, December 2014, pp. 486-491.

[17] S. Basagni, L. Bölöni, P. Gjanci, C. Petrioli, C. A. Phillips, and D. Turgut, "Maximizing the value of sensed information in underwater wireless sensor networks via an autonomous underwater vehicle," in Proc. of IEEE INFOCOM'14, April 2014, pp. 988-996.

[18] C. J. Watkins and P. Dayan, "Q-learning," Machine learning, vol. 8, no. 3-4, pp. 279-292, May 1992

[19] J. Ćosić, P. Ćurković, J. Kasać, and J. Stepanić, "Interpreting development of unmanned aerial vehicles using systems thinking," Interdisciplinary Description of Complex Systems, vol. 11, no. 1, pp. 143-152, 2013. 